\title{
Approximate homomorphisms and derivations on random Banach algebras
}

\author{
M Madadian' ${ }^{1}$ A Ebadian², M Eshaghi Gordji ${ }^{3^{*}}$ and H Azadi Kenary ${ }^{4}$
}

\section{"Correspondence:}

madjid.eshaghi@gmail.com

${ }^{3}$ Department of Mathematics,

Semnan University, P. O. Box

35195-363, Semnan, Iran

Full list of author information is

available at the end of the article

\begin{abstract}
The motivation of this paper is to investigate the stability of homomorphisms and derivations on random Banach algebras.

MSC: Primary 39B82; Secondary 39B52

Keywords: random normed algebra; generalized Hyers-Ulam stability; random homomorphism; random derivation; generalized additive functional equation
\end{abstract}

\section{Introduction and preliminaries}

The study of stability problems originated from a famous talk Under what condition does there exist a homomorphism near an approximate homomorphism? given by S. M. Ulam [38] in 1940. Next year, in 1941, D. H. Hyers [15] answered affirmatively the question of Ulam for additive mappings between Banach spaces.

Aoki [3] and Rassias [26] provided a generalization of the Hyers theorem for additive and linear functions respectively, by allowing the Cauchy difference to be unbounded.

Theorem 1.1 (Th. M. Rassias) Let $X$ be a normed space, $Y$ be a Banach space and $f: X \rightarrow$ $Y$ be a function such that

$$
\|f(x+y)-f(x)-f(y)\| \leq \varepsilon\left(\|x\|^{p}+\|y\|^{p}\right)
$$

for all $x, y \in X$, where $\varepsilon$ and $p$ are constants with $\varepsilon>0$ and $p<1$. Then there exists a unique additive function $A: X \rightarrow Y$ satisfying

$$
\|f(x)-A(x)\| \leq \varepsilon\|x\|^{p} /\left(1-2^{p-1}\right)
$$

for all $x \in X$. If $p<0$, then the inequality (1.1) holds for $x, y \neq 0$ and (1.2) for $x \neq 0$. Also, if for each fixed $x \in X$ the function $t \mapsto f(t x)$ is continuous in $t \in \mathbb{R}$, then $A$ is linear.

The above theorem had a lot of influence on the development of the generalization of the Hyers-Ulam stability concept during the last three decades. This new concept is known as generalized Hyers-Ulam stability or Hyers-Ulam-Rassias stability of functional equations (see [7, 16]). Furthermore, Gǎvruta [13] provided a generalization of Rassias' theorem which allows the Cauchy difference to be controlled by a general unbounded function. 
During the last three decades, a number of papers and research monographs have been published on various generalizations and applications of the generalized Hyers-Ulam stability to a number of functional equations and mappings (see $[4,6,8,9,12,17-19,24]$ and [27-34]). We also refer the readers to the books $[1,7,10,16,20,21,28]$.

Recently, Khodaei and Rassias [22] introduced the generalized additive functional equation

$$
\begin{aligned}
& \sum_{k=2}^{n}\left(\sum_{i_{1}=2}^{k} \sum_{i_{2}=i_{1}+1}^{k+1} \ldots \sum_{i_{n-k+1}=i_{n-k+1}}^{n}\right) f\left(\sum_{i=1, i \neq i_{1}, \ldots, i_{n-k+1}}^{n} a_{i} x_{i}-\sum_{r=1}^{n-k+1} a_{i_{r}} x_{i_{r}}\right) \\
& +f\left(\sum_{i=1}^{n} a_{i} x_{i}\right)=2^{n-1} a_{1} f\left(x_{1}\right),
\end{aligned}
$$

where $a_{1}, \ldots, a_{n} \in \mathbb{Z} \backslash\{0\}$ with $a_{1} \neq \pm 1$, and they established a general solution and the generalized Hyers-Ulam stability for the functional equation (1.3) in various spaces. They proved that a function $f$ between real vector spaces $X$ and $Y$ is a solution of (1.3) if and only if $f$ is additive.

In the sequel we adopt the usual terminology, notations and conventions of the theory of random normed spaces, as in $[36,37]$. Throughout this paper, let $\Delta^{+}$be the space of distribution functions, that is,

$$
\begin{aligned}
\Delta^{+}:= & \{F: \mathbb{R} \cup\{-\infty, \infty\} \rightarrow[0,1]: F \text { is left-continuous, } \\
& \text { non-decreasing on } \mathbb{R}, F(0)=0 \text { and } F(+\infty)=1\}
\end{aligned}
$$

and the subset $D^{+} \subseteq \Delta^{+}$is the set

$$
D^{+}=\left\{F \in \Delta^{+}: l^{-} F(+\infty)=1\right\},
$$

where, $l^{-} f(x)$ denotes the left limit of the function $f$ at the point $x$. The space $\Delta^{+}$is partially ordered by the usual point-wise ordering of functions, i.e., $F \leq G$ if and only if $F(t) \leq G(t)$ for all $t \in \mathbb{R}$. The maximal element for $\Delta^{+}$in this order is the distribution function given by

$$
\varepsilon_{0}(t)= \begin{cases}0, & \text { if } t \leq 0 \\ 1, & \text { if } t>0\end{cases}
$$

Definition $1.2([36])$ A function $T:[0,1] \times[0,1] \rightarrow[0,1]$ is a continuous triangular norm (briefly, a $t$-norm) if $T$ satisfies the following conditions:

(a) $T$ is commutative and associative;

(b) $T$ is continuous;

(c) $T(a, 1)=a$ for all $a \in[0,1]$;

(d) $T(a, b) \leq T(c, d)$ whenever $a \leq c$ and $b \leq d$ for all $a, b, c, d \in[0,1]$.

Typical examples of continuous $t$-norms are $T_{P}(a, b)=a b, T_{M}(a, b)=\min (a, b)$ and $T_{L}(a, b)=\max (a+b-1,0)$ (the Lukasiewicz $t$-norm). 
Recall (see [14]) that if $T$ is a $t$-norm and $\left\{x_{n}\right\}$ is a given sequence of numbers in $[0,1]$, $T_{i=1}^{n} x_{i}$ is defined recurrently by

$$
T_{i=1}^{n} x_{i}= \begin{cases}x_{1}, & \text { if } n=1 \\ T\left(T_{i=1}^{n-1} x_{i}, x_{n}\right), & \text { if } n \geq 2 .\end{cases}
$$

$T_{i=n}^{\infty} x_{i}$ is defined as $T_{i=1}^{\infty} x_{n+i}$.

It is known [14] that for the Łukasiewicz $t$-norm the following implication holds:

$$
\lim _{n \rightarrow \infty}\left(T_{L}\right)_{i=1}^{\infty} x_{n+i}=1 \quad \Longleftrightarrow \sum_{n=1}^{\infty}\left(1-x_{n}\right)<\infty
$$

Definition 1.3 ([37]) A random normed space (briefly, $\mathrm{RN}$-space) is a triple $(X, \mu, T)$, where $X$ is a vector space, $T$ is a continuous $t$-norm, and $\mu$ is a function from $X$ into $D^{+}$such that, the following conditions hold:

(RN1) $\mu_{x}(t)=\varepsilon_{0}(t)$ for all $t>0$ if and only if $x=0$;

(RN2) $\mu_{\alpha x}(t)=\mu_{x}\left(\frac{t}{|\alpha|}\right)$ for all $x \in X, \alpha \neq 0$;

(RN3) $\mu_{x+y}(t+s) \geq T\left(\mu_{x}(t), \mu_{y}(s)\right)$ for all $x, y \in X$ and $t, s \geq 0$.

Definition 1.4 Let $(X, \mu, T)$ be a RN-space.

(1) A sequence $\left\{x_{n}\right\}$ in $X$ is said to be convergent to $x$ in $X$ if, for every $\epsilon>0$ and $\lambda>0$, there exists a positive integer $N$ such that $\mu_{x_{n}-x}(\epsilon)>1-\lambda$ whenever $n \geq N$.

(2) A sequence $\left\{x_{n}\right\}$ in $X$ is called Cauchy if, for every $\epsilon>0$ and $\lambda>0$, there exists a positive integer $N$ such that $\mu_{x_{n}-x_{m}}(\epsilon)>1-\lambda$ whenever $n \geq m \geq N$.

(3) A RN-space $(X, \mu, T)$ is said to be complete if and only if every Cauchy sequence in $X$ is convergent to a point in $X$. A complete $\mathrm{RN}$-space is said to be a random Banach space.

Theorem 1.5 ([36]) If $(X, \mu, T)$ is a RN-space and $\left\{x_{n}\right\}$ is a sequence such that $x_{n} \rightarrow x$, then $\lim _{n \rightarrow \infty} \mu_{x_{n}}(t)=\mu_{x}(t)$ almost everywhere.

Definition 1.6 A random normed algebra is a random normed space with algebraic structure such that (RN4) $\mu_{x y}(t s)=\mu_{x}(t) \mu_{y}(s)$ for all $x, y \in X$ and all $t, s>0$.

Definition 1.7 Let $(X, \mu, T)$ and $(Y, \mu, T)$ be random normed algebras:

(i) An additive mapping $H: X \rightarrow Y$ is called a random homomorphism if $H(x y)=H(x) H(y)$ for all $x, y \in X$.

(ii) An additive mapping $D: X \rightarrow Y$ is called a random derivation if $D(x y)=D(x) y-x D(y)$ for all $x, y \in X$.

The theory of random normed spaces is important as a generalization of the deterministic result of linear normed spaces and also in the study of random operator equations. The random normed spaces may also provide us with the appropriate tools to study the geometry of nuclear physics and have an important application in quantum particle physics. The generalized Hyers-Ulam stability of different functional equations in random and fuzzy normed spaces and random and fuzzy normed algebras has been recently studied in Alsina 
[2], Miheț et al. [23], Baktash et al. [5], Saadati et al. [35], Gordji et al. [11], and Park et al. [25].

In this paper, we prove the generalized Hyers-Ulam stability of random homomorphisms and random derivations associated with the generalized additive functional equation (1.3) in random Banach algebras.

\section{Main results}

We use the following abbreviation for a given function $f$ :

$$
\begin{aligned}
D f\left(x_{1}, \ldots, x_{n}, a, b\right)= & \sum_{k=2}^{n}\left(\sum_{i_{1}=2}^{k} \sum_{i_{2}=i_{1}+1}^{k+1} \ldots \sum_{i_{n-k+1}=i_{n-k}+1}^{n}\right) f\left(\sum_{i=1, i \neq i_{1}, \ldots, i_{n-k+1}}^{n} a_{i} x_{i}-\sum_{r=1}^{n-k+1} a_{i_{r}} x_{i_{r}}\right) \\
& +f\left(\sum_{i=1}^{n} a_{i} x_{i}\right)-2^{n-1} a_{1} f\left(x_{1}\right)+f(a b)-f(a) f(b) .
\end{aligned}
$$

Theorem 2.1 Let $X$ be a real algebra, $(Y, \Lambda, T)$ be a random Banach algebra and $\xi$ : $X^{n+2} \rightarrow D^{+}\left(n \in \mathbb{N}, n \geq 2\right.$ and $\xi\left(x_{1}, \ldots, x_{n}, a, b\right)$ denoted by $\left.\xi_{x_{1}, \ldots, x_{n}, a, b}\right)$ be a function such that

$$
\lim _{m \rightarrow \infty} \xi_{a_{1}^{m} x_{1}, \ldots, a_{1}^{m} x_{n}, a_{1}^{m} a, a_{1}^{m} b}\left(\left|a_{1}\right|^{m} t\right)=1
$$

for all $x_{1}, \ldots, x_{n}, a, b \in X, t>0$ and

$$
\lim _{m \rightarrow \infty} T_{\ell=1}^{\infty}\left(\xi_{a_{1}^{m+\ell-1} x, 0, \ldots, 0}\left(2^{n-\ell-1}\left|a_{1}\right|^{m+\ell-1} t\right)\right)=1
$$

for all $x \in X$ and all $t>0$. Suppose that $f: X \rightarrow Y$ is a function satisfying

$$
\Lambda_{D f\left(x_{1}, \ldots, x_{n}, a, b\right)}(t) \geq \xi_{x_{1}, \ldots, x_{n}, a, b}(t)
$$

for all $x_{1}, \ldots, x_{n}, a, b \in X$ and $t>0$. Then there exists a unique homomorphism $H: X \rightarrow Y$ such that

$$
\Lambda_{f(x)-H(x)}(t) \geq T_{\ell=1}^{\infty}\left(\xi_{a_{1}^{\ell-1} x, 0, \ldots, 0}\left(2^{n-\ell-1}\left|a_{1}\right|^{\ell} t\right)\right)
$$

for all $x \in X$ and $t>0$.

Proof Putting $x_{1}=x$ and $a=b=x_{i}=0(i=2, \ldots, n)$ in (2.3), we obtain that

$$
\Lambda_{\left(\sum_{k=2}^{n}\left(\sum_{i_{1}=2}^{k} \sum_{i_{2}=i_{1}+1}^{k+1} \cdots \sum_{i_{n-k+1}=i_{n-k}+1}^{n} f\left(a_{1} x\right)+f\left(a_{1} x\right)-2^{n-1} a_{1} f(x)\right)\right.}(t) \geq \xi_{x, 0, \ldots, 0}(t)
$$

for all $x \in X$ and $t>0$, that is,

$$
\Lambda_{\left(\left(\begin{array}{c}
n-1 \\
n-1
\end{array}\right)+\left(\begin{array}{c}
n-1 \\
n-2
\end{array}\right)+\cdots+\left(\begin{array}{c}
n-1 \\
1
\end{array}\right)+1\right) f\left(a_{1} x\right)-2^{n-1} a_{1} f(x)}(t) \geq \xi_{x, 0, \ldots, 0}(t)
$$

for all $x \in X$ and $t>0$. It follows from the last inequality that

$$
\Lambda_{\left(1+\sum_{\ell=1}^{n-1}\left(\begin{array}{c}
n-1 \\
\ell
\end{array}\right)\right) f\left(a_{1} x\right)-2^{n-1} a_{1} f(x)}(t) \geq \xi_{x, 0, \ldots, 0}(t)
$$


for all $x \in X$ and $t>0$; hence by using the relation $1+\sum_{\ell=1}^{n-1}\left(\begin{array}{c}n-1 \\ \ell\end{array}\right)=2^{n-1}$, we have

$$
\Lambda_{2^{n-1} f\left(a_{1} x\right)-2^{n-1} a_{1} f(x)}(t) \geq \xi_{x, 0, \ldots, 0}(t)
$$

for all $x \in X$ and $t>0$. So we have

$$
\Lambda_{\frac{f\left(a_{1}^{m} x\right)}{a_{1}^{m}-f(x)}}(t) \geq T_{\ell=1}^{m}\left(\xi_{a_{1}^{\ell-1} x, 0, \ldots, 0}\left(2^{n-\ell-1}\left|a_{1}\right|^{\ell} t\right)\right)
$$

for all $x \in X$ and $t>0$. We can show that the sequence $\left\{\frac{f\left(a_{1}^{m} x\right)}{a_{1}^{m}}\right\}$ is convergent. Therefore, one can define the function $H: X \rightarrow Y$ by

$$
H(x):=\lim _{m \rightarrow \infty} \frac{1}{a_{1}^{m}} f\left(a_{1}^{m} x\right)
$$

for all $x \in X$. Now, if we put $a=b=0$, and replace $x_{1}, \ldots, x_{n}$ with $a_{1}^{m} x_{1}, \ldots, a_{1}^{m} x_{n}$ in (2.3) respectively, it follows that

$$
\Lambda_{\frac{D f\left(a_{1}^{m} x_{1}, \ldots, a_{1}^{m} x_{n}, 0,0\right)}{a_{1}^{m}}}(t) \geq \xi_{a_{1}^{m} x_{1}, \ldots, a_{1}^{m} x_{n}, 0,0}\left(\left|a_{1}\right|^{m} t\right)
$$

for all $x_{1}, \ldots, x_{n} \in X$ and all $t>0$. By letting $m \rightarrow \infty$ in (2.5), we have $D H\left(x_{1}, \ldots, x_{n}, 0,0\right)=0$; thus $H$ satisfies (1.3). Hence the function $H: X \rightarrow Y$ is additive (see also [22]). For the uniqueness property of $H$, see paper [22].

Finally, we show that $H$ is multiplicative. Since $H\left(a_{1}^{m} x\right)=a_{1}^{m} H(x)$ for all $x \in X$ and $m \in \mathbb{N}$, from (2.1) it follows that

$$
\begin{aligned}
\Lambda_{H(a b)-H(a) H(b)}(t) & =\Lambda_{\frac{1}{a_{1}^{m}} H\left(a_{1}^{m} a b\right)-H(a) H(b)}(t) \\
& =\lim _{m \rightarrow \infty} \Lambda_{\frac{1}{a_{1}^{2 m}} f\left(a_{1}^{2 m} a b\right)-\frac{1}{a_{1}^{2 m}} f\left(a_{1}^{m} a\right) f\left(a_{1}^{m} b\right)}(t) \\
& =\lim _{m \rightarrow \infty} \Lambda_{\frac{D f\left(0,0, \ldots, 0, a_{1}^{m} a, a_{1}^{m} b\right)}{a_{1}^{2 m}}}(t) \\
& \geq \lim _{m \rightarrow \infty} \xi_{0,0, \ldots, 0, a_{1}^{m} a, a_{1}^{m} b}\left(\left|a_{1}\right|^{2 m} t\right) \\
& =1
\end{aligned}
$$

for all $a, b \in X$ and all $t>0$. Therefore, there exists a unique random homomorphism $H: X \rightarrow Y$ satisfying (2.4).

In the following theorem, we establish the stability of derivations on random Banach algebras. We use the following abbreviation for a given function $f$ :

$$
\begin{aligned}
\Delta f\left(x_{1}, \ldots, x_{n}, a, b\right)= & \sum_{k=2}^{n}\left(\sum_{i_{1}=2}^{k} \sum_{i_{2}=i_{1}+1}^{k+1} \ldots \sum_{i_{n-k+1}=i_{n-k}+1}^{n}\right) f\left(\sum_{i=1, i \neq i_{1}, \ldots, i_{n-k+1}}^{n} a_{i} x_{i}-\sum_{r=1}^{n-k+1} a_{i_{r}} x_{i_{r}}\right) \\
& +f\left(\sum_{i=1}^{n} a_{i} x_{i}\right)-2^{n-1} a_{1} f\left(x_{1}\right)+f(a b)-f(a) b-a f(b) .
\end{aligned}
$$


Theorem 2.2 Let $(X, \Lambda, T)$ be a random Banach algebra and $\xi: X^{n+2} \rightarrow D^{+}$be a function such that (2.1) and (2.2) hold for all $x, x_{1}, \ldots, x_{n}, a, b \in X$ and all $t>0$. Suppose that $f: X \rightarrow$ $X$ is a function satisfying

$$
\Lambda_{\Delta f\left(x_{1}, \ldots, x_{n}, a, b\right)}(t) \geq \xi_{x_{1}, \ldots, x_{n}, a, b}(t)
$$

for all $x_{1}, \ldots, x_{n}, a, b \in X$ and $t>0$. Then there exists a unique derivation $D: X \rightarrow X$ such that

$$
\Lambda_{f(x)-D(x)}(t) \geq T_{\ell=1}^{\infty}\left(\xi_{a_{1}^{\ell-1} x, 0, \ldots, 0}\left(2^{n-\ell-1}\left|a_{1}\right|^{\ell} t\right)\right)
$$

for all $x \in X$ and $t>0$.

Proof By the same reasoning as in the proof of Theorem 2.1, the sequence $\left\{\frac{f\left(a_{1}^{m} x\right)}{a_{1}^{m}}\right\}$ is convergent for all $x \in X$, and the function $D: X \rightarrow X$ defined by

$$
D(x):=\lim _{m \rightarrow \infty} \frac{1}{a_{1}^{m}} f\left(a_{1}^{m} x\right)
$$

for all $x \in X$, is a unique additive function which satisfies (2.7). We have to show that $D: X \rightarrow X$ is a derivation.

Since $D\left(a_{1}^{m} x\right)=a_{1}^{m} D(x)$ for all $x \in X$ and $m \in \mathbb{N}$, from (2.1) it follows that

$$
\begin{aligned}
\Lambda_{D(a b)-D(a) b-a D(b)}(t) & =\Lambda_{\frac{1}{a_{1}^{m}} D\left(a_{1}^{m} a b\right)-\frac{1}{a_{1}^{m}} D(a) a_{1}^{m} b-a_{1}^{m} a \frac{1}{a_{1}^{m} D(b)}}(t) \\
& =\lim _{m \rightarrow \infty} \Lambda_{\frac{1}{a_{1}^{2 m}} f\left(a_{1}^{2 m} a b\right)-\frac{1}{a_{1}^{2 m}} f\left(a_{1}^{m} a\right) a_{1}^{m} b-\frac{1}{a_{1}^{2 m}} a_{1}^{m} a f\left(a_{1}^{m} b\right)}(t) \\
& =\lim _{m \rightarrow \infty} \Lambda_{\frac{f f\left(0,0, \ldots, 0, a_{1}^{m} a, a_{1}^{m} b\right)}{a_{1}^{2 m}}}(t) \\
& \geq \lim _{m \rightarrow \infty} \xi_{0,0, \ldots, \ldots, a_{1}^{m} a, a_{1}^{m} b}\left(\left|a_{1}\right|^{2 m} t\right) \\
& =1
\end{aligned}
$$

for all $a, b \in X$ and all $t>0$. This means that $D$ is a derivation on $X$.

Competing interests

The authors declare that they have no competing interests.

\section{Author's contributions}

All authors read and approved the final manuscript.

\section{Author details}

${ }^{1}$ Department of Mathematics, Islamic Azad University, Tabriz Branch, Tabriz, Iran. ${ }^{2}$ Department of Mathematics, Payame Noor University, Tehran, Iran. ${ }^{3}$ Department of Mathematics, Semnan University, P. O. Box 35195-363, Semnan, Iran.

${ }^{4}$ Department of Mathematics, Yasouj University, 75914-353, Yasouj, Iran.

Received: 2 March 2012 Accepted: 25 June 2012 Published: 5 July 2012

\section{References}

1. Aczél, J, Dhombres, J: Functional Equations in Several Variables. Cambridge University Press, Cambridge (1989)

2. Alsina, C: On the stability of a functional equation arising in probabilistic normed spaces. In: General Inequalities, vol. 5, Oberwolfach, 1986, pp. 263-271. Birkhäuser, Basel (1987)

3. Aoki, T: On the stability of the linear transformation in Banach spaces. J. Math. Soc. Jpn. 2, 64-66 (1950) 
4. Baker, JA: The stability of certain functional equations. Proc. Am. Math. Soc. 112, 729-732 (1991)

5. Baktash, E, Cho, YJ, Jalili, M, Saadati, R, Vaezpour, SM: On the stability of cubic mappings and quadratic mappings in random normed spaces. J. Inequal. Appl. 2008, Article ID 902187 (2008)

6. Cholewa, PW: Remarks on the stability of functional equations. Aequ. Math. 27, 76-86 (1984)

7. Czerwik, P: Functional Equations and Inequalities in Several Variables. World Scientific, Singapore (2002)

8. Eshaghi Gordji, M, Khodaei, H: Solution and stability of generalized mixed type cubic, quadratic and additive functional equation in quasi-Banach spaces. Nonlinear Anal. 71, 5629-5643 (2009)

9. Eshaghi Gordji, M, Khodaei, H: On the generalized Hyers-Ulam-Rassias stability of quadratic functional equations. Abstr. Appl. Anal. 2009, Article ID 923476 (2009)

10. Eshaghi Gordji, M, Khodaei, H: Stability of Functional Equations. Lap Lambert Academic Publishing, Saarbrücken (2010)

11. Eshaghi Gordji, M, Ghobadipour, N, Najati, A, Ebadian, A: Almost Jordan homomorphisms and Jordan derivations on fuzzy Banach algebras. Proyecciones (in press)

12. Gajda, Z: On stability of additive mappings. Int. J. Math. Math. Sci. 14, 431-434 (1991)

13. Găvruta, P: A generalization of the Hyers-Ulam-Rassias stability of approximately additive mappings. J. Math. Anal. Appl. 184, 431-436 (1994)

14. Hadžić, O, Pap, E, Budincević, M: Countable extension of triangular norms and their applications to the fixed point theory in probabilistic metric spaces. Kybernetika 38(3), 363-381 (2002)

15. Hyers, DH: On the stability of the linear functional equation. Proc. Natl. Acad. Sci. USA 27, 222-224 (1941)

16. Hyers, DH, Isac, G, Rassias, TM: Stability of Functional Equations in Several Variables. Birkhäuser, Basel (1998)

17. Hyers, DH, Rassias, TM: Approximate homomorphisms. Aequ. Math. 44, 125-153 (1992)

18. Hyers, DH, Isac, G, Rassias, TM: Topics in Nonlinear Analysis and Applications. World Scientific, Singapore (1997)

19. Isac, G, Rassias, TM: Stability of $\psi$-additive mappings: applications to nonlinear analysis. Int. J. Math. Math. Sci. 19, 219-228 (1996)

20. Jung, S-M: Hyers-Ulam-Rassias Stability of Functional Equations in Mathematical Analysis. Hadronic Press, Palm Harbor (2001)

21. Jung, S-M: Hyers-Ulam-Rassias Stability of Functional Equations in Nonlinear Analysis. Springer Optimization and Its Applications, vol. 48. Springer, Heidelberg (2011)

22. Khodaei, H, Rassias, TM: Approximately generalized additive functions in several variables. Int. J. Nonlinear Anal. Appl. $1,22-41(2010)$

23. Miheț, $D$, Saadati, R, Vaezpour, SM: The stability of an additive functional equation in Menger probabilistic $\varphi$-normed spaces. Math. Slovaca 61, 817-826 (2011)

24. Park, C, Eshaghi Gordji, M, Khodaei, H: A fixed point approach to the Cauchy-Rassias stability of general Jensen type quadratic-quadratic mappings. Bull. Korean Math. Soc. 47, 987-996 (2010)

25. Park, C, Lee, JR, Shin, DY: Generalized Ulam-Hyers stability of random homomorphisms in random normed algebras associated with the Cauchy functional equation. Appl. Math. Lett. 25, 200-205 (2012)

26. Rassias, TM: On the stability of the linear mapping in Banach spaces. Proc. Am. Math. Soc. 72, 297-300 (1978)

27. Rassias, TM: New characterization of inner product spaces. Bull. Sci. Math. 108, $95-99$ (1984)

28. Rassias, TM: Functional Equations, Inequalities and Applications. Kluwer Academic, Dordrecht (2003)

29. Rassias, TM: On the stability of the quadratic functional equation and its applications. Stud. Univ. Babes-Bolyai, Math. XLIII, 89-124 (1998)

30. Rassias, TM: The problem of S. M. Ulam for approximately multiplicative mappings. J. Math. Anal. Appl. 246, 352-378 (2000)

31. Rassias, TM: On the stability of functional equations in Banach spaces. J. Math. Anal. Appl. 251, 264-284 (2000)

32. Rassias, TM: On the stability of functional equations and a problem of Ulam. Acta Appl. Math. 62, 23-130 (2000)

33. Rassias, TM, Šemrl, P: On the behaviour of mappings which do not satisfy Hyers-Ulam stability. Proc. Am. Math. Soc. 114, 989-993 (1992)

34. Rassias, TM, Šemrl, P: On the Hyers-Ulam stability of linear mappings. J. Math. Anal. Appl. 173, 325-338 (1993)

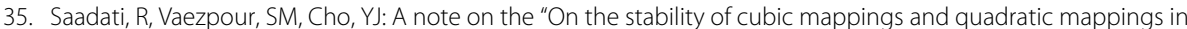
random normed spaces". J. Inequal. Appl. 2009, Article ID 214530 (2009)

36. Schweizer, B, Sklar, A: Probabilistic Metric Spaces. Elsevier, Amsterdam (1983)

37. Šerstnev, AN: On the notion of a random normed space. Dokl. Akad. Nauk SSSR 149, 280-283 (1963) (in Russian)

38. Ulam, SM: Problems in Modern Mathematics. Science Editions, Chapter VI. Wiley, New York (1964)

doi:10.1186/1029-242X-2012-157

Cite this article as: Madadian et al.: Approximate homomorphisms and derivations on random Banach algebras.

Journal of Inequalities and Applications 2012 2012:157. 